\title{
Patient safety in dentistry: Dental care risk management plan
}

\author{
Bernardo Perea-Pérez ${ }^{1}$ Andrés Santiago-Sáez ${ }^{2}$, Fernando García-Marín ${ }^{3}$, Elena Labajo-González ${ }^{4}$, Alfonso \\ Villa-Vigil ${ }^{5}$
}

\footnotetext{
${ }^{1}$ Director of the Spanish Observatory of Safety for Dentistry Patients (OESPO). Professor of the School of Legal and Forensic Medicine of Madrid. School of Medicine. Universidad Complutense de Madrid

${ }^{2}$ Member of the Spanish Observatory of Safety for Dentistry Patients (OESPO). Professor of the School of Legal and Forensic Medicine of Madrid. School of Medicine. Universidad Complutense de Madrid

${ }^{3}$ Member of the Spanish Observatory of Safety for Dentistry Patients (OESPO). Member of the Board of Directors of the Spanish Society for Oral and Maxillofacial Surgery (SECOM)

${ }^{4}$ Member of the Spanish Observatory of Safety for Dentistry Patients (OESPO). Professor of the School of Legal and Forensic Medicine of Madrid. School of Medicine. Universidad Complutense de Madrid

${ }^{5}$ President of the General Board of Dentists of Spain
}

\author{
Correspondence: \\ Escuela Medicina Legal y Forense de Madrid, \\ Facultad de Medicina. Pabellón 7, \\ Avda. Complutense $s / n$, \\ 28040. Madrid, Spain. \\ elabajo@med.ucm.es
}

Received: 04/05/2010

Accepted: 06/09/2010

Perea-Pérez B, Santiago-Sáez A, García-Marín F, Labajo-González E, Villa-Vigil A. Patient safety in dentistry: Dental care risk management plan. Med Oral Patol Oral Cir Bucal. 2011 Sep 1;16 (6):e805-9. http://www.medicinaoral.com/medoralfree01/v16i6/medoralv16i6p805.pdf

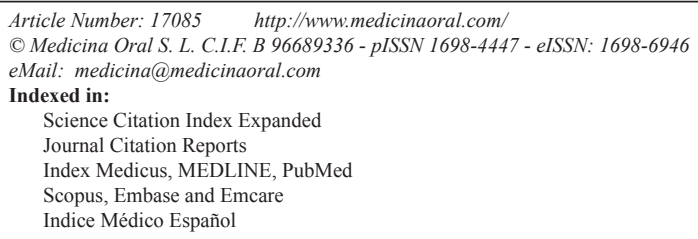

\begin{abstract}
Objectives: Although the safety of patients has been one of the inherent concerns of dental practice, but because the proposals made in the field of dentistry are few and improperly structured, this paper constitutes an attempt to present a proposal titled "Plan for Dental Health Care Risk Management," promoted by the General Council of Dentists of Spain, including a description of the proposed work methodology.

Design: The "risk management plan" proposed in this paper is based on applying the basic concepts dealt with in patient safety to the field of dentistry, due to the fact that the available bibliography contains no specific "health care risk management plan" for dentistry specifically.

Results and conclusions: In order to implement health care risk management in the field of dental care provided at any level throughout Spain, a seven-step plan which covers the main objectives in Patient Safety is provided.
\end{abstract}

Key words: Adverse events, error, clinical risk management, patient safety, oral surgery.

\section{Introduction}

Patient safety has become one of the focal points of health care in recent years. Though the concern for not harming patients (the Hippocratic "primum non nocere") has been a fundamental factor in health care since it first began, as of the publication in 1999 of the study titled "To err is human" by the Committee on Quality of Health Care in America of the Institute of Medicine, achieving safe health care practices became a top priority for health care authorities. At present, there are many institutions which have developed programs and initiatives to improve patient safety (1-3). One representative example would be the "World Alliance for the Safety of Patients" promoted by the World Health Organization 
(WHO) (4) or the Luxembourg Declaration of the European Union (5).

Within the field of dentistry, the proposals have not been as numerous or as structured. Although patient safety has also been one of the inherent concerns in dental practice, and alerts and recommendations have been given out on pharmaceutical products, dental materials and clinical procedures, the programs organized specifically for promoting patient safety have been few (6-10). At the same time, there is little structured or wellstudied data regarding adverse events in dentistry. The existing bibliography describes isolated clinical cases or short series on the basis of which empirical clinical recommendations are made.

There are reasons which explain this delay in dentistry when compared with most other health care professions: - The harm produced is generally less severe.

- The patients are ambulatory (this makes it difficult to become aware of and follow up on many adverse events).

- There is a great dispersion of dental care which makes it difficult to collect data.

- Dental care is fundamentally private, and the fear may exist that reporting adverse events might have some repercussion on the commercial profits of clinics.

- There is not a generalized culture which deals with patient safety. The motivations are different for professionals, and the potential for undertaking educational campaigns that reach all dentists is limited due to their widespread dispersion.

Nevertheless, there are many reasons why we believe that dentistry must become more active in dealing with everything involved in patient safety. Among these reasons is the fact that we handle potentially dangerous pharmaceuticals (by themselves or as a result of their interactions), dental procedures are becoming more aggressive (especially the surgical techniques related with implantology), we deal with technical instruments (ionizing radiation, lasers, etc.) which may be harmful, and the contact of our instruments with the blood and bodily fluids of patients may constitute potential sources for the transmission of diseases.

Due to all of the above, we believe that dental practice must more actively become involved in the international trend towards patient safety. It is with this goal in mind that the General Council of Dentists of Spain has promoted the "Plan for Prevention of Health Care Risks in Dentistry." The objective of this article is to explain the goals of this plan, as well as its organization and work methodology.

\section{Materials and Methods}

No available bibliography exists regarding any "plan for health care risk management" specific to dentistry. Because of this, the "risk management plan" proposed in this paper is based on applying the basic concepts dealt with in patient safety to the field of dentistry.
- Definitions.

The following definitions are provided by the ICPS (International Patient Safety Classification) proposed by the World Alliance for Patient Safety of the World Health Organization (WHO) (11).

1. Patient Safety.

Patient safety means a decrease in (or elimination of, to the greatest extent possible) the harm to patients caused by treatments provided, or from accidents associated with those treatments.

2. Risk Management.

This is the attempt to identify, evaluate and deal with problems which may cause harm to patients, to file complaints about malpractice and to avoid unnecessary economic losses for health care providers.

3. Adverse Event.

Unexpected result of a health care treatment which leads to prolonging treatment, some type of morbidity, mortality or simply any harm which the patient should not have suffered (12).

It is a broad concept which includes errors, accidents, delays in providing care, negligence, etc., but not the complications inherent to the patient's disorder or disease itself.

\section{Error.}

Mistake due to action or omission in health care practice, whether an error of planning or an error of execution (13). The error may or may not lead to the existence of an adverse event.

5. Near miss.

Event which nearly causes harm to the patient and which is avoided by luck or due to action at the last moment (14). One example would be prescribing an antibiotic derived from penicillin to an allergic patient, because this information does not appear in his or her clinical record, and gaining knowledge of that allergy because the patient informs us of it when we provide him or her with the prescription. Various studies have found that many more such incidents (near miss) occur than do actual adverse events. In the specific case of prescribing pharmaceuticals, it is estimated that approximately seven times more near misses occur than adverse events (15).

6. Accident.

Random, unforeseen and unexpected event which causes harm to the patient or any other type of harm (material damage, harm to health care personnel, etc.). 7. Negligence.

Error which is difficult to justify due to a lack of knowledge or basic skills, failure to take minimum precautions, carelessness, etc. (16).

- Professional error in dentistry.

The literature on error in medicine is extensive, especially in terms of adverse drug events, errors in prescription and medication errors, and errors in Intensive Care Units, trauma care or anesthesia (17-19). However, 
there are hardly any studies dealing with the frequency and effects of the errors committed in dental practices. This may be due to the limited repercussion on patient health which they tend to have, as well as the great dispersion of clinical records, which makes any investigation quite difficult.

The errors in dental care may be of a human origin (in which a professional reaches an erroneous decision or provides a deficient treatment), but in most cases their occurrence is to a great degree dependent upon many contributing factors related with the system, which lead to a chain of errors and end up causing harm to the patient (20).

- Oral surgery and patient safety.

Within the realm of health care, all of the research points out as the causes of the largest number of adverse events the prescription and administration of drugs, as well as surgical activity $(21,22)$. Since the advent of surgery, there has been an awareness of the potential damage which surgery may cause. The data on surgical complications occurring in industrialized nations shows that the perioperatory death rate lies between $0.4-0.8 \%$, while the rate of major complications is from $3-17 \%(21,22)$. In industrialized nations, nearly half of all adverse events are related with surgical care. Of these cases, at least half are avoidable $(21,22)$. In light of these data, in 2007 the "World Alliance for Patient Safety" (dependent upon the World Health Organization) established the "Global Patient Safety Challenge: Safe Surgery Saves Lives" as its second main objective. This change revolves around four large areas: preventing infection of the surgical wound, safe anesthesia, safe surgical equipment and the measurement of surgical services. As a part of this initiative, the "World Alliance for Patient Safety" launched the "Surgical Safety Checklist" in June 2008. This Checklist was proposed as a simple and easy-to-use tool which ensures compliance with the key patient safety key safety elements.

Amongst the specific characteristics of oral surgery which we could point out are its less invasive nature than other surgical specialties, the widespread use of local or loco-regional anesthesia, its generally ambulatory nature and the great geographic dispersion of oral health care centers. Therefore, one of the first tasks to carry out in the field of safety for patients who undergo oral surgery procedures should be the adaptation of general surgical checklists designed for major surgery in hospitals to this specific field of care.

\section{Results}

Objectives of the "Plan for Health Care Risk Prevention in Dentistry."

The general objective is to implement the management of health risks in the dental care provided at any level of care within Spanish territory.
In order to be able to achieve the Plan's general objective, the following specific goals have been established: 1. To promote a Culture of Patient Safety in dental care.

The culture of safety was defined by the ACSNI (Advisory Committee on the Safety of Nuclear Installations): "An organization's culture of safety is the product of individual and group values, attitudes, perceptions, skills and patterns of behavior which lead to commitment, style and ability in the management of the health and safety of an organization. Those organizations with a positive safety culture are characterized by communication based on mutual trust, by shared perceptions of the importance of safety and by trust in the effectiveness of measures for prevention."

2. We believe that there is a significant deficiency in terms of patient safety within the realm of health care in general, and dental care in particular. Because of this, we believe it is absolutely fundamental to promote this culture of patient safety among practicing dentists, patients and (public or private) dental care managers. To achieve this, we propose the promotion of increased awareness amongst dentists about the general aspects and basic measures involved in patient safety, as well as the main research works about this topic.

3. Creating an organizational structure for the management of dental care risks.

The manner of administrative organization in Spain, which has an independent health care system in each of its 17 autonomous regions, conditions the structure of the Observatory at two levels:

a. State-wide level: Spanish Observatory for Dental Patient Safety (OESPO).

b. Autonomous regional level: Functional units for dental patient safety dependent upon each Autonomous Regional Health Department. Each of these units would possess one representative in the OESPO, who would be the intermediary with the health care authorities of their autonomous region.

4. Developing tools for the identification, analysis and assessment of risks related with dental care.

This specific objective can, in turn, be divided into a further three:

a. Implementing a system for notification and recording of adverse events which take place in professional practice. Gaining knowledge of risk situations is basic to the implementation of any risk management system. To do so, it is necessary to have reliable notification system for any type of adverse event, which ensures the anonymity of the person who reports it. This topic seems to be of such importance to us that it will be developed as a separate specific objective.

b. Classifying the types of adverse events on the basis of a specific taxonomy. It is not useful to possess a large amount of data without first having a taxonomy that 
allows us to classify and quantify the adverse events before studying them in detail. At present, any classification should be based on the ICPS (International Patient Safety Organization) (11) or on the taxonomy proposed by the Joint Commission (23).

c. Using tools for the study of reported adverse events (root cause analysis -CRA-) and prevention of other possible adverse events (failure mode and effects analysis -FMEA-). It makes no sense to have information on adverse events if that information is not properly analyzed. In patient safety, basically two tools are used (with all of their variants): prospective and respective tools.

The classical retrospective analysis tool is root cause analysis (RCA), which would be applicable to those adverse events of sufficient significance $(24,25)$. There are other similar procedures but which are simplified for occurrences of lesser transcendence.

The most widely used prospective analysis tool is failure mode and effects analysis (FMEA). This tool uses reported data to prevent similar (potentially similar) events in similar procedures (26).

5. Establishing lines of information on adverse events. It is absolutely indispensable to possess truthful information on the occurrence of adverse events in order to be able to determine any measures for prevention. The differences existing between other health care and dental care leads to the loss of most of this information. At present only that which gives rise to legal conflicts can only be recovered, and even though only partially.

Given this situation, it is reasonable to establish different levels within these lines or sources of information: a. Bibliographic sources of information.

b. Sources of information originating from organizations similar to the OESPO (whether medical or dental).

c. Sources of information originating from ethical complaints or court claims.

d. Sources of information originating from voluntary anonymous reports made by professionals or patients (27). In this sense, the report by the Institute of Medicine (IOM) established that notification systems constitute a key strategy for learning form mistakes and avoiding their repetition.

6. Establishing measures to prevent health care risks by elimination or reduction.

These measures are to be established, on a specific basis, after identifying risks (general or specific to one center). Their purpose is the prevention of the risks identified in order to eliminate them, or if this is not possible, to reduce them.

These measures can be established at different levels:

a. At the level of a specific center, when the existence of a specific risk resulting from any particular circumstance at that center has been verified.

b. At a general level when involving known risk-producing circumstances:
I. Prevention of infections at dental offices.

II. Prevention of surgical error.

III. Prevention of error in prescribing drugs, etc.

In turn, the measures may be translated into simple recommendations or recommendations of good clinical practices. This is why it is important to analyze the experiences and recommendations of other similar entities.

7. Ongoing training of professionals on Patient Safety. This is a specific objective closely related with Objective 1 (promote a culture of Patient Safety), but it is restricted to the arena of health care professions and the care structures in which dentists perform their work.

This ongoing training should include a system of immediate alerts in the event of potentially serious cases, and the existence of a monthly bulletin in an electronic format, containing the most important information.

8. Research in the field of Dental Patient Safety.

The final specific objective, once the others have been achieved, is to increase knowledge about dental patient safety. This research may be structured around research projects, doctoral theses or any other applicable instrument of education or research.

\section{Discussion}

Patient safety constitutes a whole culture of which dental practices cannot remain on the sidelines. Nevertheless, up to the present time, few steps have been taken to bring dentistry in line with the other health care professions in this respect.

Because there are no "dental risk management plans" similar to the one proposed, its proposal is not based on any other dentistry-related documents.

Due to the breadth and complexity of the problem, we deemed it appropriate to design a plan which can be carried out sequentially and which guarantees that the objectives sought out are achieved.

The plan consists of seven steps which cover the main objectives sought in Patient Safety. Working on patient safety requires seeking humble objectives and, above all, remaining perseverant in terms of the difficulties which will inevitably arise. Through this article, we would like to offer our cooperation and share knowledge and work methodologies with any other dental organization that works to improve patient safety.

\section{References}

1. Joint Commission on Accreditation of Healthcare Organizations (JCAHO). Patient Safety Initiatives. URL http://www.jointcommission.org/PatientSafety/ [accessed on 15 May 2009].

2. National Patient Safety. URL http://www.npsa.nhs.uk/ [accessed on 15 May 2009].

3. National Patient Foundation. URL http://www.npsf.org/ [accessed on 15 May 2009].

4. World Alliance for Patient Safety. URL http:/www.who.int/patientsafety/ en/ [accessed on 16 May 2009]. 
5. EU. Luxembourg Declaration on Patient Safety. 2005. [WWW document] URL http://ec.europa.eu/health/ph_overview/Documents/ ev_20050405_rd01_en.pdf [accessed on 16 May 2009].

6. World Dental Federation (FDI). Precautions in the dental office. [WWW document] URL www.fdiworldental.org/ resources/7_0guidelines.html\#Precaution [accessed on 16 May2009]. 7. American Dental Association (ADA). URL http://www.ada.org/ public/topics/safety.asp [accessed on 17 May 2009].

8. Council of European Dentists. Patient Safety. CED Resolution May 2008. URL http://www.eudental.eu [accessed on 16 May 2009].

9. Norwegian Dental Biomaterials Adverse Reaction Unit. URL http://www.uib.no/bivirkningsgruppen/ebivirk.htm [accessed on 17 May 2009].

10. University of Sheffield. Adverse Reactions to Dental Materials. URL http://www.arrp.group.shef.ac.uk/ [accessed on 17 May 2009]. 11. Runciman W, Hibbert P, Thomson R, Van Der Schaaf T, Sherman $\mathrm{H}$, Lewalle P. Towards an International Classification for Patient Safety: key concepts and terms. Int J Qual Health Care. 2009;21:18-26.

12. Brennan TA, Leape LL, Laird NM, Hebert L, Localio AR, Lawthers AG, et al. Incidence of adverse events and negligence in hospitalized patients. Results of the Harvard Medical Practice Study I. N Engl J Med. 1991;324:370-6.

13. Hofer TP, Kerr EA, Hayward RA. What is an error? Eff Clin Pract. 2000;3:261-9.

14. Bernstein M, Herbert PC, Etchells E. Patient Safety in Neurosurgery: Detection of errors, prevention of errors, and disclosure of errors. Neurosurgery Quarterly 2003;13:125-37.

15. Bates DW, Cullen DJ, Laird N, Petersen LA, Small SD, Servi D, et al. Incidence of adverse drug events and potential adverse drug events. Implications for prevention. ADE Prevention Study Group. JAMA. 1995;274:29-34.

16. Leape LL, Brennan TA, Laird N, Lawthers AG, Localio AR, Barnes BA, et al. The nature of adverse events in hospitalized patients. Results of the Harvard Medical Practice Study II. N Engl J Med. 1991;324:377-84.

17. Vincent C, Neale G, Woloshynowych M. Adverse events in British hospitals: preliminary retrospective record review. BMJ. 2001;322:517-9.

18. Allan EL, Barker KN. Fundamentals of medication error research. Am J Hosp Pharm. 1990;47:555-71.

19. Bates DW, Boyle DL, Vander Vliet MB, Schneider J, Leape L. Relationship between medication errors and adverse drug events. J Gen Intern Med. 1995;10:199-205.

20. Hofer TP, Hayward RA. Are bad outcomes from questionable clinical decisions preventable medical errors? A case of cascade iatrogenesis. Ann Intern Med. 2002;137:327-33.

21. Weiser TG, Haynes AB, Dziekan G, Berry WR, Lipsitz SR, Gawande AA, et al. Effect of a 19-item surgical safety checklist during urgent operations in a global patient population. Ann Surg. 2010;251:976-80.

22. Greenberg CC, Roth EM, Sheridan TB, Gandhi TK, Gustafson ML, Zinner MJ, et al. Making the operating room of the future safer. Am Surg. 2006;72:1102-8.

23. Chang A, Schyve PM, Croteau RJ, O'Leary DS, Loeb JM. The JCAHO patient safety event taxonomy: a standardized terminology and classification schema for near misses and adverse events. Int $J$ Qual Health Care. 2005;17:95-105.

24. Bagian JP, Gosbee J, Lee CZ, Williams L, McKnight SD, Mannos DM. The Veterans Affairs root cause analysis system in action. Jt Comm J Qual Improv. 2002;28:531-45.

25. Ruiz-López P, González C, Alcalde-Escribano J. Análisis de causas raíz. Una herramienta útil para la prevención de errores. (Root cause analysis. A useful tool for the prevention of errors) Rev Calidad Asistencial. 2005;20:71-8.

26. Spath PL. Using failure mode and effects analysis to improve patient safety. AORN J. 2003;78:16-37.

27. Joint Commission on Accreditation of Healthcare Organizations (JCAHO). Sentinel Events Reporting Program. URL http://www.jointcommission. org/SentinelEvents [accessed on 17 May 2009]. 